\title{
CONVERGENCE OF POISSON INTEGRALS ON GENERALIZED UPPER HALF-PLANES
}

\author{
BY \\ NORMAN J. WEISS( ${ }^{(1)}$
}

1. Introduction. The object of this paper is a further generalization of the classical theorem of Fatou on the convergence of Poisson integrals on the upper half-plane and unit disk. Fatou's result states that if $f$ is an $L^{p}$ function on the line (or circle), $p \geqq 1$, and if $F$ is its Poisson integral, i.e., the convolution with $f$ of the appropriate Poisson kernel, then for almost every $x$ on the line (or circle), $F(z) \rightarrow f(x)$ as $z \rightarrow x$ nontangentially.

Generalizations in several directions have been found recently. The result has been established for arbitrary symmetric spaces in the case $p=\infty$ by Helgason and Korányi [4], for generalized upper half-planes and $p=\infty$ by Korányi and Stein [5], for symmetric spaces of rank 1 and $p \geqq 1$ by Knapp [2], and for tube domains over domains of positivity, when $f \in L^{p}, p>1$, by the author [8]. The extension of the last named result to the case $p=1$ will appear as joint work of E. M. Stein and the author (see [11], [12]).

The version of Fatou's theorem proved below is for generalized upper halfplanes (also known as Siegel domains of type II) which are holomorphically equivalent to bounded symmetric domains and functions of type $L^{p}, p>1$, or locally of type $L \log { }^{+} L$.

In $\S 2$, we sketch the setting and state our theorems explicitly. The proof, which is contained in $\S \S 3$ and 4 , is case-by-case and includes the case of the exceptional 16-dimensional domain. The technique consists of showing the Poisson integral to be bounded by a sum of maximal functions on a certain nilpotent group and establishing properties of these maximal functions by a method of rotations similar to the one used in [8].

2. The setting. Let $D$ be a generalized upper half-plane, i.e.,

$$
D=\left\{(z, w): z \in V_{1}, w \in V_{2}, \operatorname{Im} z-\Phi(w, w) \in \Omega\right\}
$$

where $V_{1}$ is a complex euclidean space of dimension $n_{1}$ with a given real form, $\Omega \subset \operatorname{Re} V_{1}$ is an open convex cone, $V_{2}$ is a complex euclidean space of dimension

Received by the editors October 31, 1967.

(1) Partially supported by the Air Force Office of Scientific Research, Harmonic Analysis Contract, at Princeton University. 
$n_{2}$, and $\Phi: V_{2} \times V_{2} \rightarrow V_{1}$ is nondegenerate and hermitian symmetric bilinear with respect to $\operatorname{Re} V_{1}$ such that $\Phi(w, w) \in \bar{\Omega}$. When $\Omega$ is a domain of positivity and $\Phi$ satisfies certain symmetry and homogeneity properties, $D$ is holomorphically equivalent to a bounded symmetric domain [6].

The distinguished boundary of $D$ is

$$
B=\{(z, w): \operatorname{Im} z-\Phi(w, w)=0\} .
$$

We identify $B$ with $\operatorname{Re} V_{1} \times V_{2}$ by identifying $(x+i \Phi(w, w), w)$ with $(x, w)$. (Despite the similarity of notation, little confusion should arise.) The measure $d \beta$ on $B$ induced by the product of the euclidean measures on $\operatorname{Re} V_{1}$ and $V_{2}$ is invariant with respect to the group $\mathfrak{R}$. $\mathfrak{R}$ is a nilpotent group of automorphisms of $D$ which acts transitively on $B$, and is also identified as a set with $\operatorname{Re} V_{1} \times V_{2}$, acting by the rule

$$
(a, c):(z, w) \rightarrow(z+a+2 i \Phi(w, c)+i \Phi(c, c), w+c) .
$$

The group product on $\mathfrak{R}$ is thus given by

$$
(a, c) \cdot\left(a^{\prime}, c^{\prime}\right)=\left(a+a^{\prime}+2 \operatorname{Im} \Phi\left(c, c^{\prime}\right), c+c^{\prime}\right),
$$

and Haar measure on $\mathfrak{R}$ is the product of the respective euclidean measures.

The Szegö and Poisson kernels are defined, respectively, on $D \times D$ and $B \times D$, and are given in the case when $\Omega$ is a domain of positivity by the formulas

$$
\begin{gathered}
S\left((z, w),\left(z^{\prime}, w^{\prime}\right)\right)=C\left[N\left(\left(z-z^{\prime}\right) / i-2 \Phi\left(w, w^{\prime}\right)\right)\right]^{-1-n_{2} / n_{1}} \\
P(u, \zeta)=P_{\zeta}(u)=|S(u, \zeta)|^{2} / S(\zeta, \zeta)
\end{gathered}
$$

where $N(z)$ is the Koecher norm function on $V_{1}$ corresponding to $\Omega$.

Finally, if $f \in L^{p}(B, \beta), p \geqq 1$, we define the Poisson integral $F$ of $f$ by

$$
F(\zeta)=\int_{B} f(u) P_{\zeta}(u) d \beta(u)
$$

The Poisson integral reproduces the real parts of holomorphic functions from their boundary values; also, Poisson integrals of arbitrary functions are harmonic in the sense of being annihilated by all invariant differential operators. Moreover, $P_{\zeta}(u)$ is an approximation of the identity on $B$, and so convergence of $F$ to $f$ in the norm is immediate. For details of the above, see [3].

We are now ready to state our main result. For $t \in \Omega, u \in B$, we write $u_{t}=u$ $+(i t, 0), F_{t}(u)=F\left(u_{t}\right)$. Let $I$ be the base point in $\Omega$.

THEOREM 2.1. Let $D$ be a generalized upper half-plane holomorphically equivalent to a bounded symmetric domain. Suppose that $f \in L^{p}(B, \beta), p>1$, or that $f \in L \log { }^{+} L$ locally and is bounded off a bounded set.

Then $\lim _{\tau \rightarrow 0} F_{\tau I}(u) \rightarrow f(u)$ for almost every $u \in B$. 
Convergence occurs even if the approach of $\zeta \in D$ to $u \in B$ is allowed to be more general. We say that $t \rightarrow 0$ restrictedly if $t$ is constrained to lie in a proper subcone of $\Omega$. Also, there is a notion which reduces to that of nontangential convergence in the case when $D$ is a tube domain, i.e., when $V_{2}=\{0\}$.

Definition 2.1. For $(a, c) \in \mathfrak{R}, \quad|(a, c)|=\max \left\{|a|,|c|^{2}\right\}$. For $\alpha>0, \Gamma_{\alpha}(0)=$ $\{g \cdot(i t, 0):|g|<\alpha|t|\}$. For $u^{0}=g_{0} \cdot 0 \in B, \Gamma_{\alpha}\left(u^{0}\right)=g_{0} \cdot \Gamma_{\alpha}(0)$.

We say that $\zeta \rightarrow u^{0}$ admissibly [5] if $\zeta$ converges to $u^{0}$ within some $\Gamma_{\alpha}\left(u^{0}\right)$. If also $t \rightarrow 0$ restrictedly, the convergence is said to be restricted admissible.

THEOREM 2.2. Under the hypotheses of Theorem 2.1, $F(\zeta) \rightarrow F(u)$ as $\zeta \rightarrow u$ restrictedly admissibly, for almost every $u \in B$.

3. The proof for domains of type $I$. Bounded symmetric domains fall into four large classes, with the exception of two exceptional domains. Those of type II, type IIIa, type IV and the exceptional domain of dimension 27 are holomorphically equivalent to tube domains over domains of positivity. Theorems 2.1 and 2.2 for these tube domains make up the content of [8].

We consider now domains of type I. There is one of these for each pair of integers $n, m, n>0, m \geqq 0$. As bounded domains, they are realized as the space of complex $n \times(n+m)$ matrices satisfying $\zeta \zeta^{*}<I$, where $I$ is the $n \times n$ identity matrix. In the realization we consider, $V_{1}$ is the complexification of the real vector space of complex hermitian symmetric $n \times n$ matrices and $V_{2}$ is the complex vector space of complex $n \times m$ matrices, so $n_{1}=n^{2}, n_{2}=m n$. Furthermore, $\Omega$ is the cone of positive definite matrices and $\Phi\left(w, w_{1}\right)=w w_{1}^{*}$. We set

$$
D=D_{n, m}=\left\{(x+i y, w): y-w w^{*}>0\right\}
$$

(In the case $m=0, D$ is a tube domain.)

Our analysis will require diagonalization of the elements of $\operatorname{Re} V_{1}$ and $V_{2}$. We recall the standard decomposition

$$
\operatorname{Re} V_{1}=E_{n} \times U(n), \quad k^{-1} d(r) k \sim(r, k),
$$

where $d(r)$ is the diagonal matrix whose entries correspond to the coordinates of the vector $r$. The above map is locally biregular except on the lower dimensional set of matrices with nondistinct eigenvalues. The map is not 1-1; it could be made so by factoring out an appropriate subgroup, but here as below, it will be more convenient not to do so. The euclidean volume element on $\operatorname{Re} V_{1}$ can be written

$$
d x=\Delta(r) d r d k, \quad \Delta(r)=c \prod_{i<j}\left(r_{i}-r_{j}\right)^{2}
$$

The analogous decomposition for $V_{2}$ depends on whether $m \leqq n$ or vice versa. 
We assume for now that the former is the case, noting that what we say can be made to hold in the latter case by exchanging $m$ and $n$. The decomposition is $V_{2}=U(n) \times E_{m} \times U(m), \quad u \tilde{d}(s) v \sim(u, s, v)$,

$$
\text { where } \tilde{d}(s)=\left(\begin{array}{cccc}
s_{1} & 0 & \cdots & 0 \\
0 & s_{2} & & \\
\vdots & & \ddots & \vdots \\
0 & & \cdots & s_{m} \\
\cdots & \cdots & 0 & \cdots
\end{array}\right)
$$

LEMMA 3.1. The euclidean volume element on $V_{2}$ can be written as

$$
\tilde{\Delta}(s) d s d u d v, \quad \tilde{\Delta}(s)=c \prod_{i<j}\left(s_{i}^{2}-s_{j}^{2}\right)^{2} \prod_{i=1}^{m} s_{i}^{2 n-2 m+1} .
$$

\section{Proof. Let}

$$
X=\left(\begin{array}{cccc}
i t_{1} & \cdots & & z_{1 n} \\
\vdots & \ddots & & \vdots \\
& & i t_{m} & z_{m n} \\
\vdots & & \vdots & \\
-\bar{z}_{1 n} & \cdots & -\bar{z}_{m n} & \cdots
\end{array}\right), \quad Y=\left(\begin{array}{ccc}
i \tau_{1} & \cdots & \zeta_{1 n} \\
\vdots & \ddots & \vdots \\
-\bar{\zeta}_{1 n} & \cdots & i \tau_{m}
\end{array}\right)
$$

$\delta s=\left(\sigma_{1}, \ldots, \sigma_{m}\right)$. Then, neglecting terms of order greater than one,

$$
\begin{aligned}
(I+X) \tilde{d}(s+\delta s)(I+Y) & =\left(\begin{array}{ccc}
s_{1}\left[1+i\left(t_{1}+\tau_{1}\right)\right]+\sigma_{1} & \\
s_{i} \zeta_{j i} & s_{i} \zeta_{i j} \\
+s_{j} z_{j i} & \ddots & +s_{j} z_{i j} \\
& & s_{m}\left[1+i\left(t_{m}+\tau_{m}\right)\right]+\sigma_{m}
\end{array}\right) \\
& =\left(\xi_{i j}+i \eta_{i j}\right) .
\end{aligned}
$$

Setting $\zeta_{i j}=\zeta_{i j}^{\prime}+i \zeta_{i j}^{\prime \prime}, z_{i j}=z_{i j}^{\prime}+i z_{i j}^{\prime \prime}$, $(i<j), T_{i}=t_{i}+\tau_{i}$, we see that the Jacobian matrix $\partial\left(\xi_{i j}, \eta_{i j}\right) / \partial\left(\zeta_{i j}^{\prime}, \zeta_{i j}^{\prime \prime}, z_{i j}^{\prime}, z_{i j}^{\prime \prime}, T_{i}, \sigma_{i}\right)$ has on its diagonal $m$ terms equal to 1 , $1+2(n-m)$ terms equal to $\pm s_{i}$ for each $i$, and 1 each of the blocks

$$
\left(\begin{array}{rr}
s_{i} & s_{j} \\
-s_{j} & -s_{i}
\end{array}\right), \quad\left(\begin{array}{ll}
s_{i} & s_{j} \\
s_{j} & s_{i}
\end{array}\right)
$$

for each $i<j$. This proves the lemma.

The next result is necessary for a detailed estimate of the Poisson kernel in certain cases. 
LEMMA 3.2. Let

$$
X=\left(\begin{array}{cccc}
0 & z_{2} & \cdots & z_{n} \\
-\bar{z}_{2} & \\
\vdots & & \cdots & \\
-\bar{z}_{n} & & &
\end{array}\right), \quad r=(\lambda, 0, \ldots, 0), \lambda>0,
$$

and $R^{2}=\left|z_{2}\right|^{2}+\cdots+\left|z_{n}\right|^{2}$.

Then $|\operatorname{det}[i d(r)+(I-X) d(r)(I+X)+I]|^{2} \geqq \lambda^{2}\left(1+\lambda^{2} R^{4}\right)$.

Proof. The matrix inside the brackets is

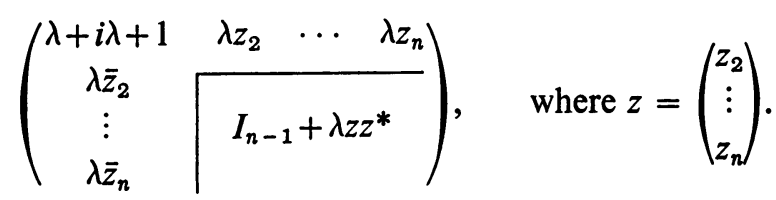

This is, except for the $i \lambda$ term, hermitian symmetric, and so

$$
\operatorname{Im} \operatorname{det}[\cdots]=\lambda \operatorname{det}\left[I_{n-1}+\lambda z z^{*}\right]=\lambda \operatorname{det}\left[d\left(1+\lambda R^{2}, 1, \ldots, 1\right)\right] .
$$

The lemma is thus proved.

We now define a class of rather complicated maximal functions on $\mathfrak{R}$, keeping in force the assumption that $m \leqq n$. The Poisson integral $F$ considered as a function on $\mathfrak{N}$, i.e., $F_{t}(g)=F_{t}(g \cdot 0), g \in \mathfrak{N}$, will be shown to be dominated by a sum of these functions. A few preliminary definitions are needed.

Let $(j)$ and $(k)$ denote, respectively, $n$-tuples and $m$-tuples of nonnegative integers such that $j_{1} \geqq \cdots \geqq j_{n}, k_{1} \geqq \cdots \geqq k_{m}$. Let $t>0$.

$$
\begin{aligned}
R_{(j)}^{t} & =\left\{r=\left(r_{1}, \ldots, r_{n}\right) \in E_{n}:\left|r_{i}\right|<2^{j} t t\right\}, \\
S_{(k)}^{t} & =\left\{s \in E_{m}:\left|s_{i}\right| \leqq 2^{k_{i}} t\right\}, \\
B_{(j)}^{t} & =\left\{x \in \operatorname{Re} V_{1}: x=k^{-1} d(r) k, r \in R_{(j)}^{t}, k \in U(n)\right\}, \\
C_{(k)}^{t} & =\left\{w \in V_{2}: w=u \tilde{d}(s) v, s \in S_{(k)}^{t}, u \in U(n), v \in U(m)\right\} .
\end{aligned}
$$

The following estimates of the measures of $B_{(j)}^{t}$ and $C_{(k)}^{t}$ will be necessary below.

LEMMA 3.3.

(a) $\left|B_{(j)}^{t}\right| \sim t^{n^{2}} 2^{(2 n-1) j_{1}+(2 n-3) j_{2}+\cdots+j_{n}}$

(b) $\left|C_{(k)}^{t}\right| \sim t^{2 n m} 2^{2\left[(n+m-1) k_{1}+(n+m-3) k_{2}+\cdots+(n-m+1) k_{m}\right]}$.

(The sign $\sim$ is taken to mean "is bounded above and below by a constant multiple of".)

Proof. The proof of (a) is contained in [8, Lemma 6.7]. The proof of (b), which amounts to showing that

$$
\int_{S_{(k)}^{t}} \tilde{\Delta}(s) d s \sim\left[\sup _{s_{(k)}^{t}} \tilde{\Delta}(s)\right]\left|S_{(k)}^{t}\right|
$$

is the same. 
Let $\mathfrak{B}_{(j)(k) l}$ be a neighborhood of the origin in $\mathfrak{u}(n)$, the Lie algebra of $U(n)$, depending on $(j),(k)$ and the nonnegative integer $l$. (The dependence will be given below.) Set $N_{(j)(k) l}=\exp \left(\mathfrak{B}_{(j)(k) l}\right)$. Finally, we define

$$
E_{(j)(k) l}^{t}=\left\{g=(x, w)=\left(k^{-1} d(r) k, u \tilde{d}(s) v\right) \in \mathfrak{R}: r \in R_{(j)}^{t}, s \in S_{(k)}^{t^{1 / 2}}, k u \in N_{(j)(k) l}\right\}
$$

(The appearance of the $t^{1 / 2}$ term is due to the quadratic term $w w^{*}$ in the Poisson kernel.)

DefinITION 3.1. Let $f$ be a function on $\mathfrak{R}$.

(a) $f_{(j)(k)}^{*}\left(x_{0}, w_{0}\right)=\sup _{t>0}\left|B_{(j)}^{t}\right|^{-1}\left|C_{(k)}^{t^{1 / 2}}\right|^{-1} \int_{B_{(j)}^{t}} \int_{C_{(k)}^{t 12}}\left|f\left(\left(x_{0}, w_{0}\right) \cdot(x, w)\right)\right| d x d w$.

$$
f_{(j)(k) l}^{*}\left(x_{0}, w_{0}\right)=\sup _{t>0}\left|E_{(j)(k) l}^{t}\right|^{-1} \int_{E_{(j)(k) l}^{t}}\left|f\left(x_{0}, w_{0}\right) \cdot(x, w)\right| d x d w
$$

The first step in the proof of Theorems 2.1 and 2.2 is to bound the Poisson integral $F_{t}(g \cdot 0)$ by a certain sum of these maximal functions evaluated at $g$. An estimate on the size of the latter will then suffice to obtain the desired result on a.e. convergence. It will be easier to proceed without stating the precise inequality that we are seeking.

First of all, let $u^{0}=g \cdot 0 \in B, g \in \mathfrak{R}$. We notice that $u_{t}^{0}=g \cdot($ it, 0$)$. By [3, Lemma 3.4], $P\left(u, u_{t}^{0}\right)=P\left(g^{-1} u,(i t, 0)\right)$, and so

$$
\begin{aligned}
F_{t}\left(u^{0}\right) & =\int_{B} f(u) P\left(u, u_{t}^{0}\right) d u=\int_{B} f(g u) P(u,(i t, 0)) d u \\
& =\int_{\mathfrak{N}} f(g h \cdot 0) P(h \cdot 0,(i t, 0)) d h .
\end{aligned}
$$

On the other hand, if $f^{*}$ is one of the maximal functions of Definition 3.1, and $f_{g}(h)=f(g h)$, then $\left(f_{g}^{*}\right)(0)=f^{*}(g)$, so it is enough to prove the inequality we seek at the identity in $\mathfrak{R}$.

For $D=D_{n, m}$, the Koecher norm function on $V_{1}$ is $N(z)=(\operatorname{det} z)^{n}$, and (1), $\S 2$ becomes

$$
P(u,(i t, 0))=P_{t}(u)=P_{t}(x, w)=C\left\{\frac{\operatorname{det} t}{\left|\operatorname{det}\left(x+i\left[w w^{*}+t\right]\right)\right|^{2}}\right\}^{n+m} .
$$

With the convention that $(j-1)=\left(j_{1}-1, \ldots, j_{n}-1\right)$ and that $2^{-1}=0$, we set

$$
\begin{aligned}
F_{(j)}^{t} & =B_{(j)}^{t}-B_{(j-1)}^{t} \subset \operatorname{Re} V_{1}, \\
G_{(k)}^{t} & =C_{(k)}^{t}-C_{(k-1)}^{t} \subset V_{2}, \\
H_{(j)(k)}^{t} & =F_{(j)}^{t} \times G_{(k)}^{t^{1 / 2}} \subset \Re .
\end{aligned}
$$


From here on, we assume for the sake of convenience and with no loss of generality that our functions $f$ are nonnegative. We would like to obtain an inequality of the following form, where $f(u)$ is understood to mean $f(u \cdot 0)$, etc..

$$
\begin{aligned}
\int_{H_{(j)(k)}^{t}} f(u) P_{t}(u) d u & \leqq\left[\sup _{H_{(j)(k)}^{t}} P_{t}(u)\right] \int_{H_{(j)(k)}^{t}} f(u) d u \\
& \leqq\left[\sup _{H_{(j)(k)}^{t}} P_{t}(u)\right] \int_{B_{(j)}^{t} \times C_{(k)}^{t 1 / 2}} f(u) d u \\
& \leqq\left[\sup _{H_{(j)(k)}^{t}} P_{t}(u)\right]\left|B_{(j)}^{t}\right|\left|C_{(k)}^{t / 2}\right| f_{(j)(k)}^{*}(0) \\
& \leqq C^{\prime} 2^{-[\mid\}|/ 2+| k \mid]} f_{(j)(k)}^{*}(0)
\end{aligned}
$$

where $|j|=j_{1}+\cdots+j_{n}$.

Let us examine the possibility of achieving (4) in the case of radial convergence. In this case, we abuse notation by using $t$ to denote both a positive number and the matrix $t I$.

In our subsequent estimates of the Poisson kernel, we will at several stages be appealing to Lemma 6.4 of [8]; the point of this result is, roughly, that $|\operatorname{det}(x+i y)|$, where $x$ is hermitian symmetric and $y$ positive definite, acts like the ordinary absolute value $|a+i b|, b>0$. We also use the fact that $t=t I$, so that $t$ and $w w^{*}$ can be diagonalized simultaneously.

By $[8,6.4]$, we can consider separately the contributions of the $x$ term and the $w w^{*}+t$ term in (3), and so

$$
\sup _{H_{(j) k(k)}^{t}} P_{t}(x, w) \leqq\left\{\begin{array}{l}
2^{-2(n+m)|j|} t^{-n(n+m)} C, \\
2^{-4(n+m)|k|} t^{-n(n+m)} C .
\end{array}\right.
$$

On the other hand, Lemma 3.3 yields the inequalities

$$
\begin{aligned}
\left|B_{(j)}^{t}\right| & \leqq 2^{(2 n-1)|j|} t^{n^{2}} C^{\prime \prime}, \\
\left|C_{(k)}^{t^{112}}\right| & \leqq 2^{2(m+n-1)|k|} t^{m n} C^{m} .
\end{aligned}
$$

In particular, (4) holds whenever

$$
|k| \geqq \frac{4 n-1}{4 n+4 m+2}|j| \text { or }|j| \geqq \frac{4 m+4 n-2}{4 m+1}|k| .
$$

The case $|k|=\frac{1}{2}|j|$ is typical of the situation when neither of these last inequalities hold. In this case, we have a common estimate,

$$
\sup _{H_{(j)(k)}^{t}} P_{t}(u) \leqq 2^{-2(n+m)|j|} t^{-n(n+m)} C,
$$

while $\left|B_{(j)}^{t}\right|\left|C_{(k)}^{t^{112}}\right| \leqq 2^{(3 n+m-2)|f|} t^{n(n+m)} C^{\prime \prime} C^{m}$, and so for (4) to hold it is necessary that

$$
2(n+m)-(3 n+m-2) \geqq 1 \text {, i.e., } n-m \leqq 1 .
$$


In order to improve our estimates in the case $m<n-1$, we must examine the joint contribution of $x$ and $w$ to $\left|\operatorname{det}\left(x+i\left[w w^{*}+t\right]\right)\right|$. As will be apparent shortly, it is enough to consider the case $(j)=(j, 0, \ldots, 0),(k)=(j / 2,0, \ldots, 0)$. Also, by homogeneity, we may assume that $t=1$.

To recapitulate, $x$ and $w w^{*}$ are hermitian symmetric $n \times n$ matrices, each having one eigenvalue between $2^{j-1}$ and $2^{j}$ in absolute value and the rest less than 1 . If, typically, $x=k^{-1} d\left(2^{j}, 0, \ldots, 0\right) k, w w^{*}=u d\left(2^{j}, 0, \ldots, 0\right) u^{-1}$, then the least amount of "interaction" occurs when $k u=I$, in which case

$$
\left|\operatorname{det}\left(x+i\left[w w^{*}+I\right]\right)\right|^{2}=2^{2 j}+\left(2^{j}+1\right)^{2} .
$$

On the other hand, if $k u$ is away from the origin in $U(n)$, the value of $|\operatorname{det}(\cdots)|^{2}$ can be as much as $\left(2^{j}+1\right)^{2}\left(2^{2 j}+1\right)$.

For a more quantitative view of the situation, we apply Lemma 3.2, noticing that $(k u)^{-1} d\left(2^{j}, 0, \ldots, 0\right)(k u)$ depends only on the coset of $k u$ modulo $S^{1} \times U(n-1)$. It follows that

$$
\left|\operatorname{det}\left(x+i\left[w w^{*}+I\right]\right)\right|^{2} \geqq 2^{2 j}\left(1+2^{2 j} R^{4}\right)
$$

whenever $k u=\exp X, X=\left(z_{i j}\right)$ and $\left|z_{12}\right|^{2}+\cdots+\left|z_{1 n}\right|^{2}=R^{2}$.

This estimate enables us to dominate $\int_{H_{(j)(k)}^{1}} f(u) P_{1}(u) d u$ by a finite sum, $2^{-j} \sum_{l=0}^{L} f_{(j)(k) l}^{*}(0)$, where $L$ depends only on $m$ and $n$.

Referring to Definition 3.1 , we see that in order to define $f_{(j)(k) l}^{*}$, we must specify $\mathfrak{B}_{(j)(k) l} \subset \mathfrak{U}(n)$. In the present situation, $(j)=(j, 0, \ldots, 0), k=(j / 2,0, \ldots, 0)$ and we set

$$
\mathfrak{V}_{(j)(k) l}=\left\{\left(z_{i j}\right):\left|z_{12}\right|^{2}+\cdots+\left|z_{1 n}\right|^{2} \leqq R_{(j)(k) l}^{2}\right\} .
$$

The decreasing numbers $R_{(j)(k) l}=R_{l}, l=0, \ldots, L$ will be defined inductively. We set

$$
\begin{aligned}
K_{(j)(k) l}^{t}= & K_{l}^{t}=\left[E_{(j)(k) l-1}^{t}-E_{(j)(k) l}^{t}\right] \cap H_{(j)(k)}^{t} \\
=\left\{g=(x, w)=\left(k^{-1} d(r) k, u \tilde{d}(s) v\right)\right. & \in \mathfrak{R}: r \in R_{(j)}^{t}-R_{(j-1)}^{t}, \\
& \left.s \in S_{(k)}^{t^{1 / 2}}-S_{(k-1)}^{t^{1 / 2}}, k u \in N_{(j)(k) l-1}-N_{(j)(k) l}\right\} .
\end{aligned}
$$

The object now is to obtain an inequality like (4), i.e.,

$$
\begin{aligned}
\int_{K_{l}^{1}} f(u) P_{1}(u) d u & \leqq\left[\sup _{K_{l}^{1}} P_{1}(u)\right] \int_{K_{l}^{1}} f(u) d u \leqq\left[\sup _{K_{l}^{1}} P_{1}(u)\right] \int_{E_{(j)(k) l-1}^{1}} f(u) d u \\
& \leqq\left[\sup _{K_{l}^{1}} P_{1}(u)\right]\left|E_{(j)(k) l-1}^{1}\right| f_{(j)(k) l-1}^{*}(0) \leqq C^{\prime} 2^{-[|j| / 2+|k|]} f_{(j)(k) l-1}^{*}(0)
\end{aligned}
$$

In the case at hand, it follows from (5) that

$$
\sup _{K_{l}^{1}} P_{1}(u) \leqq C\left[2^{-2 j}\left(1+2^{2 j} R_{l}^{4}\right)^{-1}\right]^{n+m}
$$

while on the other hand, we have from Lemma 3.3 that

$$
\left|E_{(j)(k) l-1}^{1}\right|=\left|B_{(j)}^{1}\right|\left|C_{(k)}^{1}\right|\left|N_{(j)(k) l-1}\right| \leqq C_{0} 2^{(2 n-1) j} 2^{(m+n-1) j} R_{l-1}^{2(n-1)}
$$

if $R_{l-1}$ is sufficiently small. 
We choose $R_{0}$ in such a way that $N_{(j)(k) 0}$ is all of $U(n)$. Then we choose $R_{1}<R_{0}$ large enough to make (6) hold in the case $l=1$, and continue the process. As we shall see, after $L$ steps $R_{l}$ can be taken equal to zero and the process halted. It should be emphasized that while $R_{1}=R_{j, 1} \rightarrow 0$ as $j \rightarrow \infty$, the number $L$ depends only on $m$ and $n$.

Combining (6), (7) and (8), we see that the equations determining the induction are, neglecting multiplicative constants,

$$
2^{-4(n+m) j} R_{1}^{-4(n+m)} 2^{(3 n+m-2) j}<2^{-j}
$$

and

$$
2^{-4(n+m) j} R_{l}^{-4(n+m)} 2^{(3 n+m-2) j} R_{l-1}^{2(n-1)}<2^{-j}, \quad l>1 .
$$

From this it follows that we can take $R_{1}=2^{-[(n+3 m+1) / 4(n+m)] j}$ and $R_{l}=$ $R_{1} R_{l-1}^{(n-1) / 2(n+m)}, l>1$. The $R_{l}$ converges to a positive limit, $R_{\infty}=2^{-\alpha j}$, where

$$
\alpha=\frac{1}{1-(n-1) /(2(n+m))} \cdot \frac{n+3 m+1}{4(n+m)}=\frac{n+3 m+1}{2(n+2 m+1)}>\frac{1}{2} \text {. }
$$

On the other hand, for us to take $R_{L}=0$ in (6), we must have

$$
2^{-2(n+m) j} 2^{(3 n+m-2) j} R_{L-1}^{2(n-1)}<2^{-j},
$$

or

$$
R_{L-1}<2^{-[(n-m-1) / 2(n-1)] j} .
$$

But $(n-m-1) / 2(n-1)<\frac{1}{2}$, i.e., we can take $R_{L-1}>2^{-j / 2}$, and so we can in fact stop the process after a finite number of steps, a number independent of $j$.

In general, the neighborhood $\mathfrak{B}_{(j)(k) l}$ of the origin in $\mathfrak{u}(n)$ will depend on the size of the first $m$ rows of the $\left(z_{i j}\right)$. But a few crude calculations are sufficient to show that the situation we have examined in detail, with $(j)=(j, 0, \ldots, 0)$, $(k)=(j / 2,0, \ldots, 0)$ is the worst possible. (As soon as $x$ or $w w^{*}$ has more than one eigenvalue, there must be some "interaction," in the sense described above.)

Finally, noticing that

$$
\int_{B} P_{t}(u) f(u) d u=\sum_{(j),(k), l} \int_{K_{(j)(k) l}^{t}} P_{t}(u) f(u) d u,
$$

we can bring together our calculations in

Proposition 3.4.

$$
\sup _{t>0} F\left((u \cdot 0)_{t}\right) \leqq \sum_{(j),(k)} 2^{-[|j| / 2+|k|]} \sum_{l=1}^{L_{(j)(k)}} f_{(j)(k) l}^{*}(u),
$$

where $L_{(j)(k)} \leqq L$ and $L$ depends only on $m$ and $n$.

We remark here that the criterion $\left(k^{-1} d(r) k, u \tilde{d}(s) v\right) \in E_{(j)(k) l}^{t}$ in the definition of $f_{(j)(k) l}^{*}$ is independent of a particular choice of $k$ and $u$, as is easily seen. Also, we mention that 3.4 holds equally well in the case $m \geqq n$; in fact, when $m \geqq n$, only the 
$f_{(j)(k)}^{*}$ are needed. Since the estimate of Lemma 3.3(b) is symmetric in $m$ and $n$, as is the formula (3), the same inequalities are still sufficient to establish (4), i.e.,

$$
|k| \geqq \frac{4 n-1}{4 n+4 m+2}|j| \text { or }|j| \geqq \frac{4 m+4 n-2}{4 m+1}|k| \text {. }
$$

One or the other always holds if $(4 n-1) /(4 n+4 m+2)<(4 m+1) /(4 m+4 n-2)$, which is indeed the case when $m \geqq n$.

We turn now to the maximal functions $f_{(j)(k) l}^{*}$. The situation is slightly neater in the case when $f \in L^{p}(B, \beta), p>1$. Considering $f$ as a function on $\mathfrak{R}$ in the usual way, we have

Proposition 3.5. $\left\|f_{(j)(k) l}^{*}\right\|_{p} \leqq A_{p}\|f\|_{p}$, where $A_{p}$ is independent of $(j)(k)$ and $l$.

\section{Proof.}

$f_{(j)(k) l}^{*}(g)=f^{*}(g)$

$=\sup _{t>0}\left[\frac{\int_{U(m)} \int_{U(n)} \int_{U(n)} \int_{R_{(j)}^{t}} \int_{s_{(k)}^{t / 2}} f\left(g \cdot\left(k^{-1} d(r) k, u \tilde{d}(s) v\right)\right) \chi(k u) \Delta(r) \tilde{\Delta}(s) d s d r d k d u d v}{\iiint \int \chi \chi(k u) \Delta(r) \tilde{\Delta}(s) d s d r d k d u d v}\right]$

where $\chi$ is the characteristic function of $N_{(j)(k) l}$.

Recalling from Lemma 3.3 that $\int_{R} \Delta(r) d r \sim[\sup \Delta(r)]|R|$ and similarly for $\tilde{\Delta}(s)$, we have

$$
f^{*}(g) \leqq A \frac{\int_{U(m)} \int_{U(n)} \int_{U(n)} \bar{f}(g ; k, u, v) \chi(k u) d k d u d v}{\iiint \chi(k u) d k d u d v}
$$

where

$$
\begin{aligned}
\bar{f}(g ; k, u, v) & =\bar{f}_{(j)(k)}(g ; k, u, v) \\
& =\sup _{t>0}\left|R_{(j)}^{t}\right|^{-1}\left|S_{(k)}^{t^{1 / 2}}\right|^{-1} \int_{R} \int_{S} f\left(g \cdot\left(k^{-1} d(r) k, u \tilde{d}(s) v\right)\right) d s d r .
\end{aligned}
$$

The function $\bar{f}$ may be thought of as giving maximal averages over $m+n$ dimensional rectangles "pointed" in the direction determined by $u, v$ and $k$.

Let $\mathfrak{S}_{k, u, v}=\left\{h=\left(k^{-1} d(r) k, u \tilde{d}(s) v\right): r \in E_{n}, s \in E_{m}\right\}$. By the multiplication rule of $\S 2$,

$$
\left(k^{-1} d(r) k, u \tilde{d}(s) v\right) \cdot\left(k^{-1} d\left(r^{\prime}\right) k, u \tilde{d}\left(s^{\prime}\right) v\right)=\left(k^{-1} d\left(r+r^{\prime}\right) k, u \tilde{d}\left(s+s^{\prime}\right) v\right),
$$

since $\operatorname{Im}\left[(u \tilde{d}(s) v)\left(u \tilde{d}\left(s^{\prime}\right) v\right)^{*}\right]=0$, so $\mathfrak{H}_{k, u, v}$ is a subgroup of $\mathfrak{R}$ isomorphic to $E_{n} \times E_{m}$.

It thus follows that $\bar{f}(\cdot ; k, u, v)$ restricted to the coset $g \cdot \mathfrak{S}_{k, u, v}$ is a rectangular maximal function in $E_{n} \times E_{m}$. By known results about such maximal functions (see [10, II, p. 310], where it is shown that no complications are added even though some sides of the rectangle go to zero with $t$ and others with $t^{1 / 2}$ ), we have

$$
\int_{\mathfrak{S} k, u, v}|\bar{f}(g h ; k, u, v)|^{p} d h \leqq B_{p} \int_{\mathfrak{S}_{k, u, v}}|f(g h)|^{p} d h .
$$


Moreover, since $\mathfrak{N}$ is nilpotent there is an $\mathfrak{R}$-invariant measure on the coset space $\mathfrak{N} / \mathfrak{S}[1$, p. 369]. We can thus integrate over $\mathfrak{N} / \mathfrak{S}$ on both sides of (10), concluding that

$$
\int_{\mathfrak{R}}|\bar{f}(g ; k, u, v)|^{p} d g \leqq B_{p} \int_{\mathfrak{N}}|f(g)|^{p} d g
$$

Finally, an application of the integral Minkowski inequality to (9) completes the proof of the proposition.

COROLlaRY 3.6. Suppose that $f$ is supported on a bounded subset $S$ of $\mathfrak{R}$. Then

$$
\int_{S}\left|f_{(j)(k) l}^{*}(g)\right| d g \leqq A_{1} \int_{S}|f(g)| \log ^{+}|f(g)| d g+A_{1} .
$$

Proof. The proof is that of 3.5, together with the result for rectangular maximal functions analogous to 3.6. (See [8, Lemma 3.3].)

A weak-type estimate of the form

$$
\left|\left\{g: f^{*}(g)>s\right\}\right|<A s^{-1}\|f\|_{1}
$$

would yield Theorems 2.1 and 2.2 in the case $p=1$. But weak-type inequalities cannot be "rotated" the way norm inequalities can, and so further analysis is needed. This analysis has been performed by E. M. Stein and the author; it will appear separately. (See [12].)

Proof of Theorem 2.1. The proof is a routine consequence of 3.4, 3.5 and 3.6. The method is to regard $f$ as the sum of a continuous function and a function of small $L^{p}$ (or $L \log ^{+} L$ ) norm, and then apply 3.4 and 3.5 or 3.6 to the second summand. See $[8, \S 4]$ for details.

We go on to consideration of restricted admissible convergence in two stages. First, we show that $F\left(u_{t}^{0}\right) \rightarrow f\left(u^{0}\right)$ a.e. as $t \rightarrow 0$ when $t$ is not assumed to be a scalar multiple of $I$, but is assumed to approach 0 restrictedly, i.e., within a proper subcone of $\Omega$. To prove convergence in this case, it is enough to show that if $\bar{t}>0$ is the smallest eigenvalue of $t$, then $P_{t}(u) \leqq A P_{t I}(u)$ for all $u$. This last inequality follows from [8, Lemma 6.4].

The second step is to consider restricted admissible convergence.

Lemma 3.7. Let $\zeta=g_{0} g \cdot(i t, 0) \in \Gamma_{\alpha}\left(u^{0}\right), u^{0}=g_{0} \cdot 0$. Then $P(u, \zeta) \leqq A_{\alpha} P\left(u, u_{t}^{0}\right)$ for all $u \in B$.

Proof. It is clear from the remarks above that we may take $t=t I$ as before. Also, since $P(h u, h \zeta)=P(u, \zeta), h \in \mathfrak{N}$, it is enough to consider the case $u=0$. If $g_{0}=(x, w), g=(a, c)$, then by hypothesis $\max \left\{|a|,|c|^{2}\right\}<\alpha t$, and

$$
\zeta=(x, w) \cdot\left(a+i\left[c c^{*}+t\right], c\right)=\left(\xi+i\left[(c+w)(c+w)^{*}+t\right], c+w\right),
$$

where $\xi=x+a+i\left[c w^{*}-w c^{*}\right]$. It therefore follows from (1) $\S 2$ that it suffices to prove that

$$
\left|\operatorname{det}\left(\xi+i\left[(c+w)(c+w)^{*}+t\right]\right)\right| \geqq A_{\alpha}\left|\operatorname{det}\left(x+i\left[w w^{*}+t\right]\right)\right| .
$$


We establish (11) in two steps. First we show that for arbitrary $\xi \in V_{1}$,

$$
\left|\operatorname{det}\left(\xi+i\left[(c+w)(c+w)^{*}+t\right]\right)\right| \geqq B_{\alpha}\left|\operatorname{det}\left(\xi+i\left[w w^{*}+t\right]\right)\right|
$$

and then show that for our particular $\xi$,

$$
\left|\operatorname{det}\left(\xi+i\left[w w^{*}+t\right]\right)\right| \geqq C_{\alpha}\left|\operatorname{det}\left(x+i\left[w w^{*}+t\right]\right)\right| .
$$

To establish (12), we show that as positive definite matrices,

$$
(c+w)(c+w)^{*}+t \geqq b_{\alpha}\left(w w^{*}+t\right) .
$$

In fact, if $\sigma \in E_{n}$ is a unit vector, then

$$
|(c+w) \sigma|^{2}+t \geqq(|w \sigma|-|c \sigma|)^{2}+t .
$$

Now applying the inequality $|c|^{2}<\alpha t$ in the two cases $|w \sigma|>\sqrt{ }(4 \alpha t)$ and $|w \sigma|$ $<\sqrt{ }(4 \alpha t)$, we see that the RHS of (15) dominates $b_{\alpha}\left(|w \sigma|^{2}+t\right)$, and that (14) holds. The observation that (14) implies (12) involves another slight modification of [8, Lemma 6.4].

As for (13), multiplication on the left and right by $\left(w w^{*}+t\right)^{-1 / 2}$, which can be assumed to be diagonal, leads to the inequality

$$
\left|\operatorname{det}\left(x^{\prime}+a^{\prime}+b^{\prime}+i I\right)\right| \geqq B_{\alpha}\left|\operatorname{det}\left(x^{\prime}+i I\right)\right|,
$$

where $x^{\prime} \in \operatorname{Re} V_{1}$ is arbitrary, $\left|a^{\prime}\right|<\alpha$ and

$$
b_{i j}^{\prime}=\left(r_{i}^{2}+t\right)^{-1 / 2}\left(r_{i} c_{i j}-r_{j} \bar{c}_{j i}\right)\left(r_{j}^{2}+t\right)^{-1 / 2},
$$

so in particular, $\left|b_{i j}^{\prime}\right|<2 \sqrt{ } \alpha$. The inequality (11) now follows from the analogous result for tube domains [8, Lemma 7.4]. This concludes the proof of 3.7.

Proof of Theorem 2.2. The proof is an immediate consequence of Theorem 2.1 together with the remarks above on restricted convergence and Lemma 3.7.

4. Completion of the proof. The remaining large class of domains (type IIIb) is indexed by the positive integers. As bounded domains, these are realized as the space of $2 n+1 \times 2 n+1$ skew-symmetric complex matrices satisfying $-\zeta \zeta^{*}<I$. (Type IIIa domains, spaces of $2 n \times 2 n$ matrices, are equivalent to tube domains.) In the upper half-plane realization we consider, $V_{1}$ is the complexification of the real vector space of quaternionic hermitian symmetric $n \times n$ matrices, $V_{2}$ is the complex vector space of $n$-long quaternionic vectors, and so $n_{1}=2 n^{2}-n, n_{2}=2 n$. The bilinear form is $\Phi\left(w, w_{1}\right)=w w_{1}^{*}$, where the $w$ are viewed as $n \times 1$ matrices, $\Omega \subset \operatorname{Re} V_{1}$ is the cone of positive definite matrices, and so

$$
D=D_{n}=\left\{(x+i y, w): y-w w^{*}>0\right\} .
$$

The formula (3), $\S 3$ is now

$$
P_{t}(x, w)=C\left\{\frac{\operatorname{det} t}{\left|\operatorname{det}\left(x+i\left[w w^{*}+t\right]\right)\right|^{2}}\right\}^{2 n+1} .
$$


To see that the noncommutativity of the quaternions leads to no problems, e.g., that the determinants in (17) are well defined, refer to $[9, \S 5]$. The point is that quaternionic matrices can be identified with appropriate complex matrices.

The techniques of $\S 3$ work equally well here. The analogous decompositions and magnification factors are

$$
\begin{array}{rlrl}
\operatorname{Re} V_{1} & =\operatorname{Sp}(n) \times E_{n}, & \Delta(r) & =c \prod_{i<j}\left(r_{i}-r_{j}\right)^{4}, \\
V_{2} & =\operatorname{Sp}(n) \times E_{1}, \quad \tilde{\Delta}(s)=s^{4 n-1} .
\end{array}
$$

In the terminology of the proof of Proposition 3.4, we have in place of the inequalities (7) and (8),

$$
\begin{gathered}
\sup _{K_{j l}^{1}} P_{1}(u) \leqq C\left[2^{-2 j}\left(1+2^{2 j} R_{l}^{4}\right)\right]^{2 n+1}, \\
\left|E_{j, l-1}^{1}\right|=\left|B_{j}^{1}\right|\left|C_{j / 2}^{1}\right|\left|N_{j, l-1}\right| \leqq C_{0} 2^{(4 n-3) j} 2^{2 n j} R_{l-1}^{4(n-1)} .
\end{gathered}
$$

This leads to

$$
R_{1}=2^{-[(n+3) / 2(2 n+1)]\}} \text { and } R_{\infty}=2^{-[(n+3) / 2(n+2)] j}
$$

while $R_{L-1}=2^{-[(n-2) / 2(n-1)] j}$, and so the inductive process is again a finite one.

The rest of the proof of Theorem 2.1 and 2.2 follows in the case of type IIIb domains exactly as it did for type I domains.

The remaining bounded symmetric domain which is not equivalent to a tube domain is an exceptional domain of complex dimension 16. In its realization as a generalized upper half-plane, $V_{1}=V_{1}=C_{8}$, and $\Omega$ is the forward light cone in $E_{8}$, i.e.,

$$
\Omega=\left\{\left(x_{1}, \ldots, x_{8}\right): x_{1}>\left(x_{2}^{2}+\cdots+x_{8}^{2}\right)^{1 / 2}\right\} .
$$

We can proceed in this case without knowing the bilinear form $\Phi$ explicitly. For ease of notation, we continue to write $\Phi\left(w, w_{1}\right)=w w_{1}^{*}$.

Let $D$ be the exceptional domain, $B$ its distinguished boundary, and identify $B$ with $E_{8} \times C_{8}$ as usual. For $x, y \in E_{8}$, we write

$$
[x, y]=x_{1} y_{1}-x_{2} y_{2}-\cdots-x_{8} y_{8} .
$$

In this case, the Koecher norm function is $N(z)=[z, z]^{n / 2}$, and it follows from (1), §2 that

$$
\begin{aligned}
P(u,(i t, 0)) & =P((x, w),(i t, 0))=P_{t}(x, w) \\
& =C\left\{\frac{[t, t]}{\left.\left([x, x]-\left[w w^{*}+t, w w^{*}+t\right]\right)^{2}+4\left[x, w w^{*}+t\right]^{2}\right)}\right\}^{8} .
\end{aligned}
$$

The decomposition of $\operatorname{Re} V_{1}$ is

$$
E_{8}=E_{2} \times S O(7) / S O(6)=E_{2} \times S^{6}, \quad \Delta\left(r_{1}, r_{2}\right)=\left(r_{1}-r_{2}\right)^{6},
$$

where the two real parameters correspond to $x_{1} \pm\left(x_{2}^{2}+\cdots+x_{8}^{2}\right)^{1 / 2}$. Also

$$
V_{2}=C_{8}=E_{1} \times S^{15}, \quad \tilde{\Delta}(s)=s^{15} \text {. }
$$


We show that there is an equality like (4), $\S 3$ in the exceptional case. If $x=$ $\left(x_{1}, \ldots, x_{8}\right)$, we write $\rho=\left(x_{2}^{2}+\cdots+x_{8}^{2}\right)^{1 / 2}$, and notice that $[x, x]=\left(x_{1}+\rho\right)\left(x_{1}-\rho\right)$. The sets that appear in the exceptional version of (4) are

We have

$$
\begin{aligned}
B_{(j)}^{t} & =\left\{x:\left|x_{1}\right|+\rho \leqq 2^{j_{1}} t,|| x_{1}|-\rho| \leqq 2^{j_{2}} t\right\}, \\
C_{k}^{t^{1 / 2}} & =\left\{w:|w| \leqq 2^{k} t^{1 / 2}\right\}, \\
H_{(j) k}^{t} & =\left(B_{(j)}^{t}-B_{(j-1)}^{t}\right) \times\left(C_{k}^{t^{1 / 2}}-C_{k-1}^{t^{1 / 2}}\right) .
\end{aligned}
$$

$$
\left|B_{(j)}^{t}\right|\left|C_{k}^{t^{1 / 2}}\right| \sim 2^{7 j_{1}+j_{2}+16 k} t^{16} .
$$

As before, we can dominate $P_{t}(u)$ by considering separately the contributions of $x$ and $w$, and assume that $t=(t, 0, \ldots, 0)$.

In particular,

$$
\sup _{H_{(j) k}^{t}} P_{t}(u) \leqq C 2^{-16|j|} t^{16}
$$

As for $P_{t}(0, w)$ when $|w| \sim 2^{k} t^{1 / 2}$, the worst case occurs when $w w^{*}$ has one "eigenvalue" equal to a multiple of $2^{2 k} t$ and the other equal to zero. And then

$$
P_{t}(0, w)=C\left(t^{2} /\left[w w^{*}+t, w w^{*}+t\right]^{2}\right)^{8} \leqq C^{\prime} 2^{-32 k} t^{-16} .
$$

Combining this with (18), (19) and (20), and considering separately the cases $|j| \geqq 2 k,|j| \leqq 2 k$, we obtain

$$
\left[\sup _{H_{(j) k}^{t}} P_{t}(u)\right]\left|B_{(j)}^{t}\right|\left|C_{k}^{t^{1 / 2}}\right| \leqq C^{\prime} 2^{-[|j| / 2+k]}
$$

which enables us to proceed as in $\S 3$ and complete the proof of Theorem 2.1 for the exceptional domain.

The argument of $\S 3$ proving restricted convergence a.e. goes through without change, since it is basically a tube domain result. Finally, the argument proving admissible convergence a.e. in $\$ 3$ needs only slight modification to yield the same result in the exceptional case. And thus Theorem 2.2 holds in the case of the exceptional domain.

Proofs of Theorems 2.1 and 2.2. A generalized upper half-plane equivalent to a bounded symmetric domain is the direct product of half-planes equivalent to irreducible bounded symmetric domains. But half-planes of the latter type are either tube domains over irreducible domains of positivity, for which 2.1 and 2.2 were proved in [8], or domains of the types considered in $\$ 3$ and $\S 4$ above. The usual methods [10, II, Chapter 17], [8, $\$ 7]$ for dealing with products of domains lead to the proof of 2.1 and 2.2 in their full generality.

5. Concluding remarks. The notion of admissible convergence is an extension of the euclidean one of nontangential convergence. In particular, Theorem 2.2 extends previously known results, e.g., [7], about a.e. convergence of holomorphic functions of the class $H^{p}(D)$, where $D$ is a domain of the type we have considered. 
The case-by-case method presented here lends little insight to some of the more general problems involved. For instance, the requirement that the generalized upper half-plane be equivalent to a bounded symmetric (homogeneous) domain is not crucial. On the one hand, Knapp [2] proved Fatou's theorem for all symmetric spaces of rank 1. And on the other hand, the theorem is also true for at least one upper half-plane not equivalent to a bounded symmetric domain.

In fact, we can prove 2.1 and 2.2 for the half-plane of complex dimension 4 equivalent to a bounded, homogeneous, nonsymmetric domain. In this case, $V_{1}$ is the complexification of the space of real symmetric $2 \times 2$ matrices, $\Omega$ is the cone of positive definite matrices, $V_{2}=C$, and

$$
\Phi\left(w, w_{1}\right)=\left(\begin{array}{cc}
0 & 0 \\
0 & w \bar{w}_{1}
\end{array}\right) .
$$

It is easy to establish an inequality like (4), $\S 3$, and the rest of the proof follows as it did in $\S 3$.

\section{REFERENCES}

1. S. Helgason, Differential geometry and symmetric spaces, Academic Press, New York, 1961.

2. A. Knapp, Fatou's theorem for symmetric spaces. I, Ann. of Math. (2) 88 (1968), 106-127.

3. A. Korányi, The Poisson integral for generalized half-planes and bounded symmetric domains, Ann. of Math. (2) 82 (1965), 332-350.

4. A. Korányi and S. Helgason, A Fatou-type theorem for harmonic functions on symmetric spaces, Bull. Amer. Math. Soc. 74 (1968), 258-263.

5. A. Korányi and E. M. Stein, Fatou's theorem for generalized halfplanes, C.I.M.E. summer course on bounded homogeneous domains, Cremonese, 1967.

6. A. Korányi and J. Wolf, Realization of hermitian symmetric spaces as generalized halfplanes, Ann. of Math. (2) 81 (1965), 265-288.

7. E. M. Stein, Note on the boundary values of holomorphic functions, Ann. of Math. (2) 82 (1965), 351-353.

8. N. J. Weiss, Almost everywhere convergence of Poisson integrals on tube domains over cones, Trans. Amer. Math. Soc. 129 (1967), 283-307.

9. - Almost everywhere convergence of Poisson integrals on tube domains over cones, Thesis, Princeton Univ., Princeton, N. J., 1966.

10. A. Zygmund, Trigonometric series, 2nd ed., Cambridge Univ. Press, New York, 1959.

11. E. M. Stein, Maximal functions and Fatou's theorem, C.I.M.E. summer course on bounded homogeneous domains, Cremonese, 1967.

12. E. M. Stein and N. J. Weiss, Convergence of Poisson integrals for bounded symmetric domains, Proc. Nat. Acad. Sci. U.S.A. 60 (1968), 1160-1162.

Princeton University, Princeton, New Jersey 